\title{
Lamellar Arrangement in Facelift Procedures
}

\author{
KARIMA T. ISMAIL, M.D. and MARIAM T. ISMAIL, M.D. \\ The Department of Plastic and Reconstructive Surgery, Faculty of Medicine, Cairo University
}

\begin{abstract}
Introduction: The aging process of the face involves: Skin elastosis, lipodystrophy, gravitational effect and atrophy. For decades while planning facelift procedures, the face was divided into regions (upper, middle, lower face). Reflecting on the etiology of aging it seems logic to plan facelift procedures in horizontal fashion rather than traditional vertical planning. In this study we are addressing the face in 3 lamellas: Skin, subcutaneous fat and SMAS where we are combining the advantages of both techniques: Traditional supra-SMAS and deep composite facelift.
\end{abstract}

Material and Methods: Retrospective study from 2014 to 2017 included 52 cases. Procedures were done using sedation and local anesthesia. Incisions followed by skin undermining (1 $1^{\text {st }}$ lamella) and ligament release from superficial fat compartments and SMAS. Thus, allowing repositioning of superficial fat compartments (2nd lamella, e.g. malar pad of fat) and SMAS plication in upward vector; in which composite facelift lacks. Followed by sub-SMAS and sub-platysma dissection ( $3^{\text {rd }}$ lamella) giving advantage of platysma redraping over the neck, enabling good pull and mobilization of SMAS (re-enhancing our previous fat repositioning and plication) and allowing SMAS excision (preventing lateral cheek bulge). Plication, excision and SMAS anchoring. Skin and SMAS pull in upward and lateral vector. Ancillary procedures as chin augmentation and fat injection were used when needed.

Results: Follow-up from 6 months to 2 years. $84.6 \%$ complied to follow-up, $90.9 \%$ were satisfied, chin implant in $9 \%$, lipo-injection in $22.7 \%$. Complications $13.6 \%$, early: $6.8 \%$ had tenderness and redness managed by antibiotic ointment, $2.2 \%$ had hematoma collection which was surgically evacuated and coagulation of bleeding vessels, $2.2 \%$ had mandibular neuropraxia for 3 weeks; late: $6.8 \%$ hypertrophic scarring.

Conclusion: Restoring the harmonious facial anatomy during facelift procedure is crucial step. Lamellar facelift ensures addressing all facial components as a safe, reliable procedure that ensures long term desired aesthetic outcome.

Key Words: Facelift-Aging - Elastosis.

\section{INTRODUCTION}

The aging process of the face consists of intrinsic and extrinsic processes. The intrinsic process includes:

1- Elastosis of the skin, thinner epidermis with flattening of the dermal-epidermal junction.
2- Lipodystrophy and accumulation of fat in different areas of face and neck.

3- Atrophy of bone, fat and maybe muscles.

4- Gravitational effect that causes tissue descent [1].

The extrinsic process is caused primarily by sun exposure causing photoaging. Characterized by wrinkles, dryness, irregular pigmentation, telangiectasia, purpura and loss of elasticity [2].

Facial proportions and proper facial analysis play an important role in incision placement and operative technique. Midface width, facial length and facial fullness are the most important facial proportions that are put into consideration. During planning of face lift procedures, we usually think of the face as regions: Upper face, middle face, lower face and neck [3,4]. However, reflecting on the etiology of aging it seems logic to plan face lift procedures in horizontal fashion (lameller) rather than the traditional vertical planning. In this study we are addressing the face in 3 lamellas: Skin, subcutaneous fat and SMAS where we are combining the advantages of both techniques: Traditional supra-SMAS and deep composite facelift.

\section{MATERIAL AND METHODS}

Retrospective study from 2014 to 2017 included 52 cases. Procedures were done using sedation and local anesthesia. In this study we are addressing the face in three lamellae: Skin, subcutaneous fat and SMAS. The first lamella is skin which folds by undermining, ligament release and traction. Fine wrinkles are treated by laser post operatively.

The second lamella is subcutaneous fat which is managed by liposuction, lipo-injection, dissection and elevation by sutures to specific fat pads namely malar fat pad andjowls. Leveling of fat lobules by multiple 4/0 vicryl sutures.

The third lamella is SMAS, which is addressed by plication, excision and SMAS anchoring (2 flap 
suspension technique). Platysmo-plasty and full width muscle transection maybe accompanied according to neck severity of the case.

Lamellar arrangement:

- Incision-Liposuction.

- Undermining and ligamentous release.

- Malar fat elevation.

- SMAS dissection and suspension by 2 flap technique.

- Jowl elevation.

- Leveling of the surface by multiple small $4 / 0$ vicryl sutures.

- Ethibond corset neck sutures.

- Skin pull in upward and lateral vectors.

\section{RESULTS}

Follow-up period varied from 6 months to 2 years. $84.6 \%$ of the patients complied to followup, $90.9 \%$ were satisfied, chin implant was done

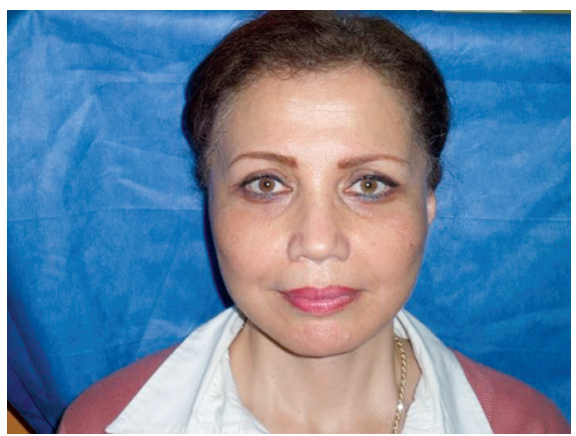

Fig. (1B): Post-operative: 6 months. in $9 \%$, lipo-injection was done in $22.7 \%$. Complications rate was $13.6 \%$, varied from early complications: $6.8 \%$ had tenderness and redness managed by antibiotic ointment, $2.2 \%$ had hematoma collection which was surgically evacuated and coagulation of bleeding vessels, $2.2 \%$ had mandibular neuropraxia for 3 weeks; late complication was $6.8 \%$ hypertrophic scarring.

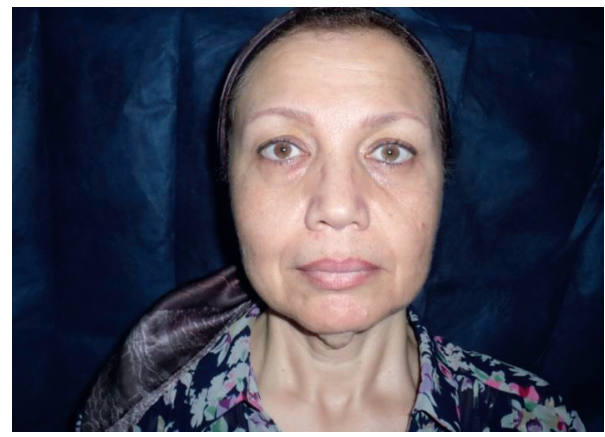

Fig. (1A): Pre-operative.

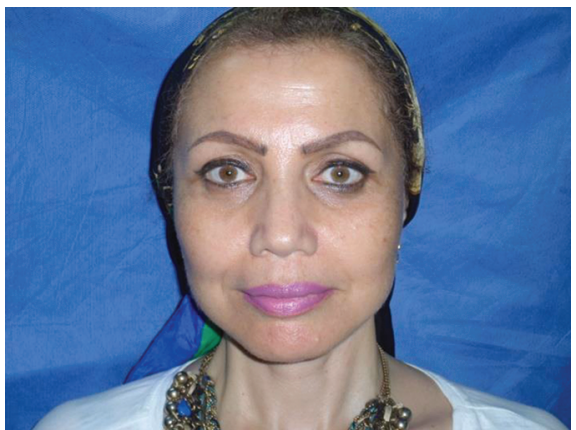

Fig. (1C): Post-operative: 15 months.

After lamellar facelift and lipoinjection.

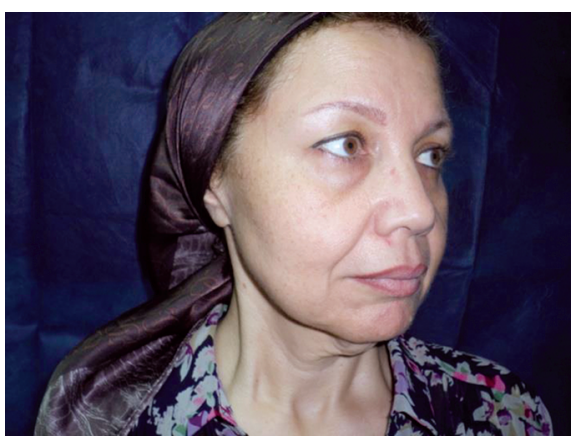

Fig. (2A): Pre-operative.

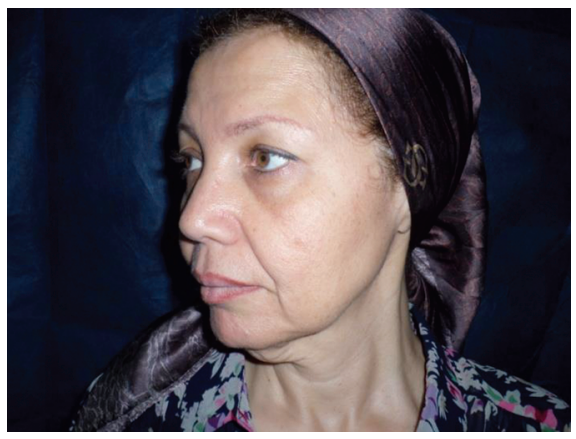

Fig. (3A): Pre-operative.

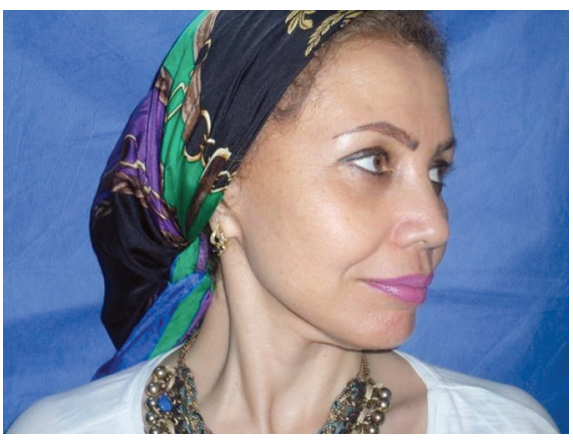

Fig. (2B): 15 months post-operative.

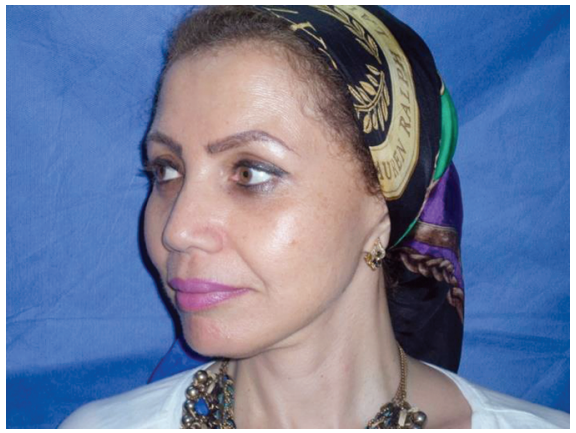

Fig. (3B): 15 months post-operative. 


\section{DISCUSSION}

The first lamella: Skin is affected by aging process through elastosis and photoaging. Therefore, best treated by undermining, ligament release, traction and to be followed by laser treatment $[\mathbf{1 , 3}]$.

The second lamella is the subcutaneous fat which is affectsthe aging process by many ways. Excess fat maybe presents in Submental region (preplatysmal, subplatysmal) Submandibularregion, Pre parotid regionand Neck. Atrophy as in buccal and temporal fat pads [5]. Descent of subcutaneous fat, as for example: Malar fat pad causingnasolabial fold prominence, labio-mental fad pad cause jowl prominence [6]. Iatrogenic abnormal condensation and induration. Therefore, subcutaneous fat manipulation must vary from liposuction, lipo-injection, dissection and elevation by sutures to specific fat pads namely malar fat pad andjowls. Leveling of fat lobules by multiple 4/0 vicryl sutures.

The third lamella is SMAS which descent by the effect of gravity and neck bands (platysmal bands) are found in some cases. Therefore, it is addressed by plication, excision and SMAS anchoring. Platysmo-plasty and full width muscle transection maybe accompanied according to neck severity of the case $[\mathbf{7 , 8}]$.

\section{Conclusion:}

Restoring the harmonious facial anatomy during facelift procedure is a crucial step. Lamellar facelift allows arranging layers of the face and individual manipulation of each layer addressing all facial components resulting in a safe and reliable procedure that ensures long term desired aesthetic outcome.

\section{REFERENCES}

1- Khavkin J. and Ellis D.A.: Aging skin: Histology, physiology, and pathology. Facial Plast Surg. Clin. North Am., 19: 229-234, 2011.

2- Gonyon D.L. Jr. and Barton F.E. Jr.: The aging face: Rhytidectomy and adjunctive procedures. Sel. Read Plast. Surg., 11, 2012.

3- Nahai F. ed.: The Art of Aesthetic Surgery: Principles \& Techniques. St Louis: Quality Medical Publishing, 2005.

4- Rohrich R.J., Ghavami A., Lemmon J.A., et al.: The individualized component face lift: Developing a systematic approach to facial rejuvenation. Plast. Reconstr. Surg., 123: 1050-1063, 2009.

5- Lambros V.: Observations on periorbital and midface aging. Plast. Reconstr. Surg., 120: 1367-1376, 2007.

6- Rohrich R.J., Pessa J.E. and Ristow B.: The youthful cheek and the deep medial fat compartment. Plast. Reconstr. Surg., 121: 2107-2112, 2008.

7- Hamra S.T.: Composite rhytidectomy. Plast. Reconstr. Surg., 90: 1-13, 1992.

8- Baker D.C., Aston S.J., Guy C.L., et al.: The male rhytidectomy. Plast. Reconstr. Surg., 60: 514-522, 1977. 